\title{
THIẾT KẾ HỆ THỐNG ĐIỀU KHIỀN SỐ TỐI ƯU Bî̀N VỮNG VỚI PHẢN HỒI ĐẦU RA
}

\author{
CHU VĂN HÝ
}

\begin{abstract}
This paper considers the problem of designing quadratic optimal digital control systems with uncertain parameters. For the output feedback systems a parametric optimization technique is used. A method for computing the supremum of a quadratic forme is proposed. The stability of the closed-loop systems is also discussed.
\end{abstract}

\section{I. ĐẶT VẤN Đ产}

Để giải quyết vấn đề bất định của hệ thống, tiểp theo các lý thuyết điều khiển ngẫu nhiên, tự thích nghi... hiện nay điều khiển bền vững (Robust Control) đang được quan tâm đặc biệt. Trong điều khiển ngẫu nhiên, ta giả thiết biết trước xác xuất của các thông số bất định. Trong điều khiển tự thích nghi, tính chất thống kê của các thông số không biết trước nhưng được nhận dạng trong qua trình điều khiển. Còn trong điều khiển bền vững: ta giả thiết các thông số biến đối trong một khoàng cho trước xung quanh giá trị danh đinh.

Trong trường hợp hệ thống được mô tả bằng phương trình trạng thái, bài toán điều khiển tối ưu bền vững các hệ thống liên tục đã có một số phương pháp hữu hiệu $[1],[2],[3] \ldots$ Nhưng cho các hệ thống thời gian rời rạc, kết quá còn rất ít. Trong bài này, chúng tôi trình bày một phương pháp thiết kế hệ thống điều khiển số nhiều đầu vào nhiều đầu ra chứa bất định trong cả ma trận động lực và ma trận đầu vào. Cho hệ thống với phản hồi đầu ra, một kĩ thuật tối ưu hóa thông số thích hợp đã được sứ dụng. Một phương pháp tính giá trị lớn nhất (Supremum) cúa dạng toàn phương - vấn đề có tính đặc trưng của bài toán điều khiển tối ưu bền vững - được dẫn ra. Tính chất ổn định của hệ thống kín cũng được bàn đến.

\section{II. ĐIỀU KHIỄN TỐI UU BỀN VŨ̃NG}

\section{Thành lập bài toán}

Xét hệ thống điều khiền số tuyến tính 


$$
\begin{aligned}
x(k+1) & =A(q) x(k)+B(q) u(k) \\
y(k) & =C x(k)
\end{aligned}
$$

Trong đó: $x(k) \in R^{n}$ là vectơ trạng thái, $u(k) \in R^{r}$ là vectơ điều khiền, $y(k) \in R^{m}$ là vectơ đầu mơ rộng: gồm các đầu ra và một số trạng thái đo được dùng làm phán hồi. Véctơ thông số bất định $q \in R^{p}$ biến đổi trong miền khép kín bị giới hạn $\Omega$, tác động tuyến tính vào ma trận động lực và ma trận đầu vào:

$$
\begin{aligned}
& \Omega:=\left\{q \in R^{p}: a_{i} \leq q_{i} \leq b_{i} ; a_{i} \leq 0, b_{i} \geq 0, i=1,2, \ldots, p\right\} \\
& A(q)=A+\Delta A=A+\sum_{i=1}^{p} q_{i} A_{i} \\
& B(q)=B+\Delta B=B+\sum_{i=1}^{p} q_{i} B_{i}
\end{aligned}
$$

Trong đó: $A, B$ biễu diễn chế đậ danh định của hệ thống, $A_{i}, B_{i}$ là các ma trận hằng, mô tá cấu trúc của bất định. Ta cần tìm điều khiển phản hồi đầu ra.

$$
u(k)=K y(k)
$$

đễ đưa hệ thống từ trạng thái ban đầu $x(0)$ trở lại trạng thái cân bằng $x(\infty)=0$, sao cho cực tiểu hóa hàm mục tiêu

$$
I:=\sup _{q \in \Omega}\left\{\sum_{k=0}^{\infty} x^{T}(k) Q x(k)+2 x^{T}(k) S u(k)+u^{T}(k) R u(k)\right\}
$$

Trong đó các ma trận trọng: $Q=Q^{T} \geq O, R=R^{T}>0$.

\section{Lời giài tối ưu}

Trước hết, ta cần biễu diễn hàm mục tiêu $J$ theo ma trận khuếch đại $K$ phải tìm và các thông số khác của hệ thống. Thay $u(k)$ từ $(6),(2)$ vào $(7)$ ta có

$$
J=\sup _{q \in \Omega}\left\{\sum_{k=0}^{\infty} x^{T}(k) Q_{c} x(k)\right\}
$$

Trong đó:

$$
Q_{c}=Q+2 S K C+C^{T} K^{T} R K C
$$


Nhờ $(1),(6),(2)$ ta lần lượt biểu diễn $x(k), k=1,2, \ldots$ theo $x(0)$ và tính được

$$
I=\sup _{q \in \Omega}\left\{x^{T}(0)\left[\sum_{k=0}^{\infty}\left(A_{c}^{k}(q)\right)^{T} Q_{c} A_{c}^{k}(q)\right] x(0)\right\}
$$

Trong đó $A_{c}(q)$ là ma trận động lực cúa hệ thống kín

$$
A_{c}(q)=A(q)+B(q) K C
$$

Ta có giả thiết: $A_{c}(q)$ là ma trận ồn định (có các giá trị riêng nằm trong đường tròn đơn vị trên mặt phẳng phức). Vì chỉ như thế bài toán điều khiển tối ưu mới có ý nghĩa. Sau đó có thể chứng minh, [4]: chuỗi vô tận ở vế phải của (10) hội tụ tới $P_{1}$

$$
\sum_{k=0}^{\infty}\left(A_{c}^{k}(q)\right)^{T} Q_{c} A_{c}^{k}(q)=P_{1}
$$

Trong đó $P_{1}$ là nghiệm duy nhất của phương trình đại số

$$
P_{1}=A_{c}^{T}(q) P_{1} A_{c}(q)+Q_{c}
$$

Vậy, bài toán trên đây là tương đương với việc tìm ma trận $K$ để cực tiểu hóa hàm mục tiêu $J$ :

$$
J=x^{T}(0) P x(0)
$$

với ràng buộc đằng thức

$$
P=\sup _{q \in \Omega}\left\{A_{c}^{T}(q) P A_{c}(q)+Q_{c}\right\}, Q_{c}=Q_{c}^{T}>0
$$

Trong đó $P=P^{T}>0$ là giá trị lớn nhất (supremum) theo nghĩa

$$
x^{T}(0)\left\{A_{c}^{T}(q) P A_{c}(q)+Q_{c}\right\} x(0) \leq x^{T}(0) P x(0) ; \forall q \in \Omega, \forall x(0) \neq 0
$$

Ta viết (15) dưới dạng chuần

$$
F:=\sup _{q \in \Omega}\left\{A_{c}^{T}(q) P A_{c}(q)+Q_{c}\right\}-P=0
$$

Bài toán tối ưu với ràng buộc đẳng thức $(14),(17)$ có thể giải theo các phương pháp biến phân, nhân tứ Lagrange, gradient $[4]$... Trong bài này, bằng cách đưa 
vào ma trận các. nhân tứ Lagrange $L$ đối xứng (do $F$ dối xứng), ta chuyển về giải bài toán cực tiểu hóa hàm $H$ không ràng buộc

$$
\begin{gathered}
H:=x^{T}(0) P x(0)+\sum_{i=1}^{n} \sum_{j=1}^{n} L_{i j} F_{i j}=\operatorname{tr}\left[P x(0) x^{T}(0)\right]+\sum_{i=1}^{n} \sum_{j=1}^{n} L_{i j} F_{j i} \\
=\operatorname{tr}\left[P x(0) x^{T}(0)\right]+\operatorname{tr}[L F]=\operatorname{tr}\left[P x(0) x^{T}(0)+L F\right]
\end{gathered}
$$

Ta xét trường hợp thường gặp: số điều khiển bằng số đầu ra $r=m, K$ là ma trận vuông. Lời giải tối ưu có thể nhận được từ điều kiện cần: các đạo hàm riêng của $H$ theo $L, P, K$ bằng 0 :

$$
\begin{gathered}
\sup _{q \in \Omega}\left\{[A(q)+B(q) K C]^{T} P[A(q)+B(q) K C]\right\}+Q+2 S K C \\
+C^{T} K^{T} R K C-P=0 \\
\sup _{q \in \Omega}\left\{[A(q)+B(q) K C] L[A(q)+B(q) K C]^{T}\right\}+x(0) x^{T}(0)-L=0 \\
2 \sup _{q \in \Omega}\left\{B^{T}(q) P A(q) L C^{T}\right\}+2 \sup _{q \in \Omega}\left\{B^{T}(q) P B(q) K C L C^{T}\right\}+2 S^{T} L C^{T} \\
+2 R K C L C^{T}=0
\end{gathered}
$$

Thay $A(q), B(q)$ theo(4), (5) ta nhận được kết quả - sau khi biến đổi:

$$
\begin{aligned}
& P=(A+B K C)^{T} P(A+B K C)+Q+2 S K C+C^{T} K^{T} R K C+U(P, K) \\
& L=(A+B K C) L(A+B K C)^{T}+x(0) x^{T}(0)+V(L, K) \\
& K=-\left(R+B^{T} P B\right)^{-1}\left(B^{T} P A L C^{T}+S^{T} L C^{T}+W(P, L, K)\left(C L C^{T}\right)^{-1}\right.
\end{aligned}
$$

Trong đó:

$$
\begin{aligned}
U(P, K)=\sup _{q \in \Omega}\{ & 2(A+B K C)^{T} P \Delta A+\Delta A^{T} P, \Delta A+2(A+B K C)^{T} P \Delta B K C \\
& \left.+2 \Delta A^{T} P \Delta B K C+C^{T} K^{T} \Delta B^{T} P \Delta B K C\right\} \\
W(P, K, L)= & \left.\sup _{q \in \Omega}\left\{\Delta B^{T} P A+B^{T} P \Delta A+\Delta B^{T} P \Delta A\right) L C^{T}\right\} \\
+ & \sup _{q \in \Omega}\left\{\left(\Delta B^{T} P B+B^{T} P \Delta B+\Delta B^{T} P \Delta B\right) K C L C^{T}\right\}
\end{aligned}
$$


Các giá trị lớn nhất (supremum) ơ đây được hiểu tương tự như $P$ trong $(15)$ theo nghĩa ở (16). Để giải hệ phương trình $(22),(23),(24)$ ta có thể dùng phương pháp lặp tương tự trong [2], [6]. Ghi chú: (22), (23) là các phương trình Liapunov bị biến dạng do có thêm các số hạng $U(P, K), V(L, K)$.

\section{Cách tính các giá trị lớn nhất}

Phương pháp tính giá trị lớn nhất của dạng toán phương $x^{T}(0) M x(0)$ của ma trận $M$ ở đậy dựa vào đượng chéo hóa ma trận đối xứng $M^{*}$ có cùng dạng toàn phương: $x^{T}(0) \dot{M}^{*} x(0)=x^{T}(0) M x(0)$. Ta chia các ma trận trong dấu \{\} ra 2 loại:

a) Loại đơn giản: chỉ chứa tích của một ma trận khoảng, như $(A+B K C)^{T} P \triangle A$, $(A+B K C)^{T} P \triangle B K C,(A+B K C) L \Delta A^{T}, \triangle B K C L(A+K B C)^{T}, \triangle B^{T} P A L C^{T}$, $B^{T} P \triangle A L C^{T} \ldots$

b) Loại phức tạp: chứa tích của ma trận khoảng, như $\Delta A^{T} P \Delta A, \Delta A^{T} P \Delta B K C$, $\triangle A L \Delta A^{T}, \triangle B K C L \Delta A^{T}, \Delta B^{T} P \Delta A L C^{T}, \Delta B^{T} P \Delta B K C L C^{T} \ldots$

Truờng hợp a: Ví dụ ta phải tính

$$
U=\sup _{q \in \Omega}\left\{(A+B K C)^{T} P \Delta A\right\}
$$

Thay $\Delta A$ theo (4) ta có:

$$
U=\sup _{q \in \Omega}\left\{(A+B K C)^{T} P \sum_{i=1}^{p} q_{i} A_{i}\right\}=\sum_{i=1}^{p} U_{i}
$$

Trong đó:

$$
U_{i}=\sup _{q \in \Omega}\left\{q_{i}(A+B K C)^{T} P A_{i}\right\}
$$

Tức là cần tìm các ma trận $U_{i}$ sao cho

$$
x^{T}(0) q_{i}(A+B K C)^{T} P A_{i} x(0) \leq x^{T}(0) U_{i} x(0), a_{i} \leq q_{i} \leq b_{i}, \forall x(0)
$$

Kí hiệu:

$$
M_{i}=(A+B K C)^{T} P A_{i}
$$

Ta thành lập ma trện đối xứng $M_{i}^{*}$ có dạng toàn phương

$$
x^{T}(0) M_{i}^{*} x(0)=x^{T}(0) M_{i} x(0)
$$

bằng cách lấy các phần tự

$$
\left(M_{i}^{*}\right)_{k j}=\left(M_{i}^{*}\right)_{j k}=\left[\left(M_{i}\right)_{k j}+\left(M_{i}\right)_{j k}\right] / 2
$$


Ta xác định ma trận modal $N_{i}$ của $M_{i}^{*}$. Trong trường hợp $M_{i}^{*}$ là ma trận thực, đối xứng thì $N$ là ma trận trực giao, đường chéo hóa $M_{i}^{*}$ :

$$
\begin{gathered}
N_{i}^{-1} M_{i}^{*} N_{i}=\Lambda_{i} \\
N_{i}^{-1}=N_{i}^{T}, \quad N_{i} N_{i}^{T}=I
\end{gathered}
$$

Trong đó $\Lambda_{i}$ là ma trận đường chéo chứa các giá trị riêng $\left(\lambda_{i}\right)_{k}, k=1,2 \ldots, n$ của $M_{i}^{*}$. nên có thể viết

$$
\begin{aligned}
x^{T}(0) q_{i}(A+B K C)^{T} P A_{i} x(0) & =x^{T}(0) N_{i} q_{i} A_{i} N_{i}^{T} x(0)=\left(x^{*}\right)^{T} q_{i} \Lambda_{i} x^{*} \\
& =\sum_{i=1}^{n} q_{i}\left(\lambda_{i}\right)_{k}\left(x_{k}^{*}\right)^{2}
\end{aligned}
$$

Dễ dàng nhìn thấy: trong khoáng $a_{i} \leq q_{i} \leq b_{i}$, dạng bình phương (37) có giá rị nhất khi:

$$
q_{i}= \begin{cases}a_{i} \ldots & \text { nếu }\left(\lambda_{i}\right)_{k}<0 \\ b_{i} \ldots & \text { nếu }\left(\lambda_{i}\right)_{k} \geq 0\end{cases}
$$

Từ đó suy ra cách tính

$$
U=\sum_{i=1}^{p} N_{i} E_{i} N_{i}^{T}
$$

Trong đó $E_{i}$ là ma trận đường chéo với các phần tử

$$
\begin{aligned}
& \left(E_{i}\right)_{k k}= \begin{cases}a_{i}\left(\lambda_{i}\right)_{k} \ldots & \text { nếu }\left(\lambda_{i}\right)_{k}<0 \\
b_{i}\left(\lambda_{i}\right)_{k} \ldots & \text { nếu }\left(\lambda_{i}\right)_{k} \geq 0\end{cases} \\
& \left(E_{i}\right)_{k h}=0 ; \forall k \neq h ; k, h=1,2, \ldots, n
\end{aligned}
$$

Trường hợp b: Ví dụ

$$
V=\sup _{q \in \Omega}\left\{\triangle B K C L \Delta A^{T}\right\}
$$

Thay $\Delta A, \Delta B$ theo (4), (5) ta có:

$$
V=\sup _{q \in \Omega}\left\{\left(\sum_{i=1}^{p} q_{i} B_{i}\right) K C L\left(\sum_{j=1}^{p} q_{j} A_{j}^{T}\right)\right\}=\sum_{i=1}^{p} \sum_{j=1}^{p} V_{i j}
$$

Trong đó:

$$
V_{i j}=\sup _{q \in \Omega}\left\{q_{i} q_{j} B_{i} K C L A_{j}^{T}\right\}
$$


Nghĩa là:

$$
\begin{gathered}
x^{T}(0) q_{i} q_{j} B_{i} K C L A_{j}^{T} x(0) \leq x^{T}(0) V_{i j} x(0) \\
a_{i} \leq q_{i} \leq b_{i}, a_{j} \leq q_{j} \leq b_{j}, \forall x(0)
\end{gathered}
$$

Tương tự như trên, ta tính

$$
M_{i j}=B_{i} K C L A_{j}^{T}
$$

Sau đó tìm ma trận đối xứng $M_{i j}^{*}$ - có dạng toàn phương $x^{T}(0) M_{i j}^{*} x(0)=$ $x^{T}(0) M_{i j} x(0)$; tính các giá trị riêng $\left(\lambda_{i j}\right)_{k}, k=1,2 \ldots n$ và ma trận modal $N_{i j}$ cúa $M_{i j}$. Ta có công thức:

$$
V=\sum_{i=1}^{p} \sum_{j=1}^{p} N_{i j} E_{i j} N_{i j}^{T}
$$

Trong đó:

$$
\begin{gathered}
\left(E_{i j}\right)_{k k}= \begin{cases}m_{1}\left(\lambda_{i j}\right)_{k} \ldots & \text { nếu }\left(\lambda_{i j}\right)_{k}<0, \text { với } m_{1}=\min \left(a_{i} b_{j}, a_{j} b_{i}\right) \\
m_{2}\left(\lambda_{i j}\right)_{k} \ldots & \text { nếu }\left(\lambda_{i j}\right)_{k} \geq 0, \text { với } m_{2}=\max \left(a_{i} a_{j}, b_{i} b_{j}\right)\end{cases} \\
\left(E_{i j}\right)_{k h}=0 ; \forall k \neq h ; k, h=1,2, \ldots, n
\end{gathered}
$$

\section{III. ỔN ĐỊNH CƯA HỆ THỐNG KÍN}

Ta xét điều kiện để hệ thống tối ưu sẽ ổn định theo định nghĩa của Liapunov. Chọn hàm Liapunov có dạng

$$
V(x(k))=x^{T}(k) P_{L} x(k)
$$

Trong đó: $P_{L}=P_{L}^{T}>0$. Theo (47), (1), (6), (2) ta tính được

$$
\begin{aligned}
\Delta V & =V(x(k+1))-V(x(k)) \\
& =x^{T}(k)\left[(A(q)+B(q) K C)^{T} P_{L}(A(q)+B(q) K C)-P_{L}\right] x(k)
\end{aligned}
$$

Hệ thống kín sẽ ổn định tiệm cận $(x(\infty)=0)$, nếu tìm được ma trận $P_{L}$ sao cho:

$$
x^{T}(k)\left[(A(q)+B(q) K C)^{T} P_{L}(A(q)+B(q) K C)-P_{L}\right] x(k)<0, \forall q \in \Omega, \forall x(k) \neq 0
$$

Bởi vì (15), (16), (17), (19), (22) là tương đương, nên so sánh (49) với (16) suy ra: điều kiện (49) sẽ thỏa mãn nếu ta chọn $P_{L}=P$ cho $Q_{c}=Q_{c}^{T}>0$. Nếu $Q_{c} \geq 0$, 
hệ thống ổn định - nhưng không nhất thiết ổn định tiệm cận. Như vậy, sau khi tính ma trận khuếch đại $K$ ta xác định dấu của $Q_{c}$ theo (9) và kết luận được tính ôn định của hệ thống. Trong trường hợp $Q_{c}$ không xác định dương, cần chọn lại các ma trận $Q, S, R$. Ta thấy: tìm điều kiện cho các ma trận trọng để hệ thống tối ưu đồng thời ồn định ở đây phức tạp hơn trong các hệ thống phản hồi trạng thái. Cho trường hợp đặc biệt $S=0$, từ (9) dễ dàng nhìn thấy: $Q=Q^{T} \geq 0$, $R=R^{T}>0$ là điều kiện cần và đủ để $Q_{c}^{T}>0$ và hệ thống tối ưu là ổn định tiệm cận.

\section{KẾT LUẬN}

Ta có thể áp dụng phương pháp trên cho trường hợp tổng quát nhất - khi tất cả các ma trận của hệ thống chứa bất định: $A(q), B(q), C(q)$. Tính các giá trị lớn nhất $U(P, K), V(L, K), W(P, L, K)$ sẽ phức tạp hơn. Bời vì các phương trình $(19),(20),(21)$ chỉ là điều kiện cần, nên có thể có một6 số nghiệm không phải là tối ưu. Lúc đó, đế xác định nghiệm tối ưu cần so sánh giá trị của hàm mục tiêu trong tất cà các trường hợp.

\section{TÀI LIẾU THAM KHẢO}

1. Kosmiddou O., Bertrand P, Rubust-Controller design for systems with large parameter variations. Int. J. Contr., Vol. 45, No. 3 (1987) 927-938.

2. $\mathrm{Hu} \mathrm{H}$., Lon $\mathrm{N}$, Robust optimal parametrix $L Q$ control with guaranteed cost bound and applications. Int. J. Contr., Vol. 50, No.6 (1989) 2489-2502.

3. Douglas J., Athan M., Robust linear quadratic designs with real parameter uncertainty. IEEE Trans. on AC, Vol. 39, No. 1 (1994) 107-111.

4. Oreilly J., Newmann M.M., On the disign of discrete - time optimal dynamical controllers using a minimal-order obder observer. Int. J. Contr., Vol. 23, No. 2 (1976) 257-275.

5. Rodellar J., Leitmann G., Ryan P., Output feedback control of uncertain couped systems. Int. J. Contr., Vol. 58, No. 2 (1993) 445-457.

6. Chu Văn Hy, Diều khiển bền vững tối uu bình phuơng với phân hồi đầu ra. Tạp chí Tin học và Điều khiển học, T. 11, S. 4 (1995).

Viện Công nghê thông tin

Trung tâm KHTN và CNQG

Nhận bài ngày 14-3-1996 